\title{
A Simple Lapicque Neuron Emulator
}

\author{
Fatih Tulumbaci ${ }^{1}$, Mehmet Hamza Eryildiz ${ }^{2}$, Resat Mutlu ${ }^{3}$ \\ ${ }^{1}$ Electronics Department, Corlu Vocational School, Tekirdag Namik Kemal University \\ 59860, Corlu, Tekirdag, Turkey \\ ftulumbaci@nku.edu.tr \\ ${ }^{2}$ Electrical Installation Design Engineer, R\&D Department, Hattat Traktor \\ 59500, Cerkezkoy, Tekirdag, Turkey \\ mehmethamzaeryildiz@gmail.com \\ ${ }^{3}$ Electronics and Communication Engineering Department, Corlu Engineering Faculty, Tekirdag Namik Kemal University \\ 59860, Corlu, Tekirdag, Turkey \\ rmutlu@nku.edu.tr
}

\begin{abstract}
Neuron models such as Leaky Integrate and Fire (Lapicque Neuron) model, Hodgkin Huxley Model, Fitzhugh-Nagumo Model, and Izhikevich neuron model are commonly used in neuronal studies. Nobel laureate Hudgkin-Hugsley neuron model is quite complex despite of being accurate. That's why simplified neuron models such as Fitzhugh-Nagumo neuron model is commonly used in studies. Lapicque Neuron is the first neuron model suggested in Literature. It had been reported by Lapicque in 1907, it is easy to understand, and it is still commonly used for neural studies for its simplicity. Neuron emulator circuits are used for education and research purposes. They can be made using individual electronics components or VLSI circuits. In this study, a Lapicque Neuron emulator circuit topology is introduced. It is simple enough to be made by students and it can be made using cheap off-the shelves components in a short time. Its circuit analysis is also given. It is experimentally shown that the emulator is able to mimic activation potentials well. It is suggested that the neuron emulator can be used for not only educational purposes in Biomedical Engineering Courses but also neuronal studies to show and prove concepts.
\end{abstract}

Keywords: Lapicque Neuron, Lapicque Neuron models, neuron emulators, biomedical engineering education.

\section{Introduction}

Integrate-and-fire model of a neuron is suggested by Louis Lapicque [1]. A review article of the original Lapicque's 1907 paper can be found in [2]. Lapique neuron model paved the way for more complex Hudgin-Hugsley neuron model [34]. Since Hudgin-Hugsley neuron model is more complex, it takes more time to simulate and harder to emulate using circuits. That's why simplified neuron models such as Lapicque model, Fitzhugh-Naguma model, Izhikevich neuron model and their varities are used for studying neuron dynamics [5-11]. Although there are more developed neuron models and Lapique neuron model is being the simplest neuron model of them all, even today, Lapicque's neuron model is still one of the most used neuron models in neuroscience. It has found application areas in neural and cellular networks studies and also in computational neurology due to its simplicity [2, 12-31]. Synchronization of two cardiomyocyte with Integrate and fire neuron model model is examined in [16]. Adaptive exponential integrate-and-fire neuron model is used to show emergence of synchronised firing patterns in a random network in [17]. A fractional-order leaky integrate-and-fire neuron model is shown to have long-term memory and power law dynamics in [18]. The leaky integrate and fire neural networks are shown to produce chimera states which are coexisting patterns of synchrony and desynchrony in [19]. An adaptive neural firing pattern is studies with a variant of the integrate-and-fire neuron model in [20]. Self-synchronization of the integrate-and-fire pacemaker model with continuous couplings is examined in [21]. A network of integrate and fire neurons is used for visual selection [22]. Origin of firing varibility of the integrate-and-fire model is examined in [23,24]. An integrate-and-fire model of a cerebellar granule cell is made in [25]. Chaotic firing is shown to exist in the sinusoidally forced leaky integrate-and-fire model with threshold fatigue in [26]. Effects of passive dendritic tree properties on the firing dynamics of a leaky integrate-and-fire neuron are inspected in [27]. The parameters of an integrate and fire neuron is tuned via a genetic algorithm for solving pattern recognition problems in [28]. Sensitivity of the coefficient of variation of interspike intervals on the lower boundary of membrane potential for the leaky integrate-and-fire neuron model is studied 
in [29]. Approximate information capacity of the perfect integrate-and-fire neuron using the temporal code is estimated in [30]. Timeseries prediction ability of a single integrate-and-fire neuron is examined in [31].

Neuron emulators are commonly used to examine neuron systems or behaviour of neurons [32-40]. As shown in [1231], Lapique neuron model is quite useful and an emulator of the neuron would be quite beneficial for not only research but also educational purposes. In this study, first, Lapicque neuron model is summarized and, then a Lapicque neuron emulator circuit which can be made using cheap and off-the shelves circuit components is suggested. Experiments are done on the designed emulator circuit to evaluate its performance.

This article is organized as the follows. Lapique's neuron model is introduced in the second section. A Lapique neuron emulator circuit is given, it is also explained how it works, and its circuit is analysed in the third section. The experimental results are given in the fourth section. The article is finished with conclusion section.

\section{Lapicque's Integrate-And-Fire Model Neuron}

Louis Lapicque presented his neuron model in time domain using constitutive equation of a capacitor:

$$
I(t)=C \frac{d V(t)}{d t}
$$

where $C$ is capacitance of the neuron's membrane and $V$ is the membrane voltage.

When an input current $I$ flows through the neuron membrane, its voltage rises linearly with time until it becomes equal to a fixed threshold voltage $V_{T H}$, at that point a Dirac delta function spike happens and the membrane voltage gets reset to its resting potential. The neuron continues to integrate and fire periodically after the reset. The operating or the firing frequency of the Lapique neuron designated as $f(I)$ is proportional to its current.

$$
f(I)=\frac{I}{C V_{T H}}
$$

The firing frequency rises linearly without any limit as the membrane current increases. A refractory period tref can be used to make the Lapique neuron model more accurate by limiting its operating frequency. The firing frequency in this case given as

$$
f(I)=\frac{I}{C V_{T H}+t_{r e f} I}
$$

Although a shortcoming of this neuron model is that it does not possess a time-dependent memory. If the membrane current becomes zero and its voltage is below its threshold voltage at some time, it keeps that voltage indefinitely and it does not fire until it is fed again with a sufficient amount of current for a sufficiently long time. This retention characteristic does not fit observed neuronal behaviour.

\section{The Lapicque Neuron Emulator Circuit}

The Lapicque Neuron Emulator Circuit shown in Figure 1 is suggested in this paper. It has two opamps. One of the opamps, U1, is used as a voltage follower fed by a voltage divider made of $\mathrm{R}_{1}$ and $\mathrm{R}_{2}$ resistors to obtain the membrane resting potential. The electromechanical relay RL1 and the transistor Q1 are used to fire the neuron emulator to reset its voltage to the membrane resting potential. The transistor Q1 is triggered by the opamp U2 when the potential at its positive node becomes equal to the emulator thereshold voltage $V_{T H}$ obtained by the voltage divider made of $R_{3}$ and $R_{4}$ resistors. When this happens, the capacitor voltage becomes zero. The potential at U2's positive node is also the neuron emulator voltage. The freewheeling diode D1 is used to protect the relay RL1. 


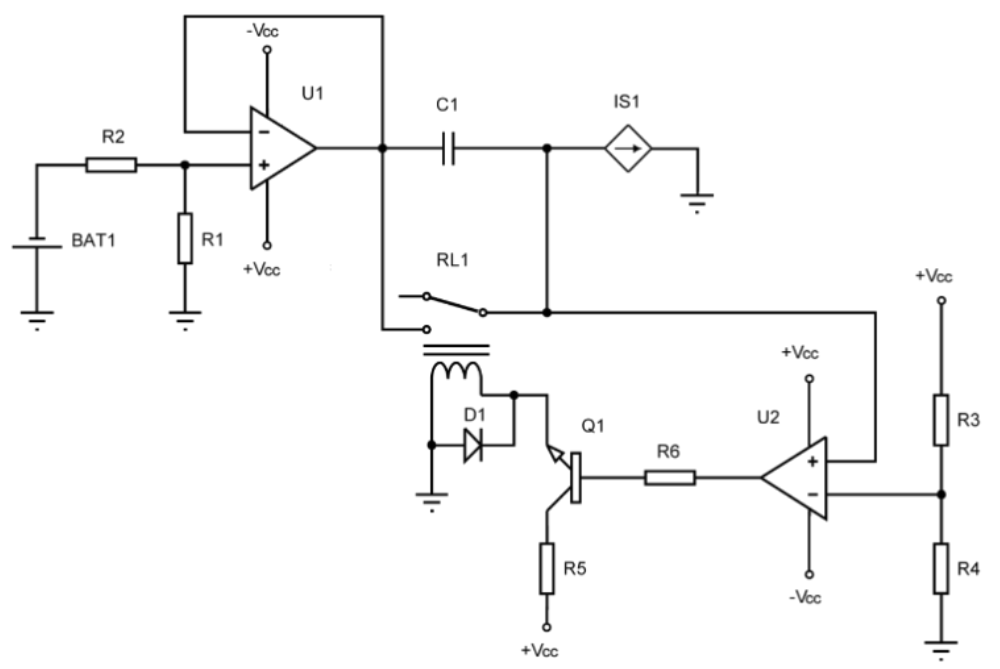

Fig. 1: Lapique neuron emulator schema.

The circuit analysis of the Lapicque neuron emulator is given as the follows. The capacitor current is given as

$$
i_{C}=C \frac{d v_{C}}{d t}
$$

Where $v_{C}$ is the capacitor current and $C$ is the membrane capacitance. The capacitor is fed by the current source $I_{S}$. Therefore, its current is given as

$$
i_{C}=I_{S}
$$

$V_{\min }$ is the membrane resting potential obtained by the voltage divider made of $R_{l}$ and $R_{2}$ resistors and it calculated as

$$
V_{\min }=-V_{c c} \frac{R_{1}}{R_{1}+R_{2}}
$$

The neuron voltage is the sum of the capacitor current and the voltage across $R_{2}$ reflected by means of using an opamp:

$$
V_{\text {neuron }}(t)=v_{C}(t)+V_{\min }
$$

Firing period of the neuron emulator $\tau$ is found as

$$
\tau=C\left(V_{\max }-V_{\min }\right) / I_{S}
$$

Where $V_{\max }$ is the threshold voltage obtained by the voltage divider made of $R_{3}$ and $R_{4}$ resistors and it calculated as

$$
V_{\text {max }}=V_{c c} \frac{R_{4}}{R_{3}+R_{4}}
$$

Firing frequency of the neuron emulator is given as

$$
f=\frac{1}{\tau}=\frac{I_{S}}{C\left(V_{\max }-V_{\min }\right)}
$$

By adjusting the current source magnitude, firing frequency of the neuron emulator can be adjusted.

\section{Experimental Results}

The Lapicque Neuron Emulator is made of the components given in Table 1. The Lapique neuron emulator circuit is made as a printed circuit board shown in Figure 2 and is used in the experiments. A digital oscilloscope is used to acquire the neuron emulator voltage and the acquired waveforms are shown in Figure 3. Due to the relay RL1 and the freewheeling diode D1 used, the circuit produces some dead time therefore the period is less than the calculated neuron firing time. The voltage waveforms have some spiking due the used relay's contact bouncing (uncontrolled opening and closing of the 
contacts due to internal forces within the relay). As shown in Figure 3, the neuron emulator mimics the activation potentials well. İnterestingly, the spikes in the voltage waveform can be interpreted as the unsuccessful or failed neuron firing.

Table 1: The Component Values

\begin{tabular}{|c|c|c|c|}
\hline \multicolumn{3}{|c|}{ Circuit Elements } \\
\hline \multicolumn{2}{|c|}{$\begin{array}{c}\text { Sources and } \\
\text { Feeds }\end{array}$} & \multicolumn{2}{c|}{ Resistors } \\
\cline { 3 - 5 } R1 & $1.8 \mathrm{~K} \Omega$ \\
\hline BAT 1 & $5 \mathrm{~V}$ & $\mathrm{R} 2$ & $12 \mathrm{~K} \Omega$ \\
\hline IS1 & $50 \mu \mathrm{A}$ & $\mathrm{R} 3$ & $33 \mathrm{~K} \Omega$ \\
\hline +Vcc & $+5 \mathrm{~V}$ & R4 & $4.7 \mathrm{~K} \Omega$ \\
\hline -Vcc & $-5 \mathrm{~V}$ & R5 & $10 \Omega$ \\
\hline \multicolumn{2}{|c|}{ Opamps } & R6 & $330 \Omega$ \\
\cline { 4 - 5 } & U1 & TLC272 & Capacitor \\
\hline U2 & TLC272 & C1 & $47 \mathrm{nF}$ \\
\hline \multicolumn{3}{|c|}{ Relay } & \multicolumn{3}{|c|}{ Diode } \\
\hline RL1 & $5 \mathrm{~V}$ & D1 & $1 \mathrm{~N} 4001$ \\
\hline
\end{tabular}
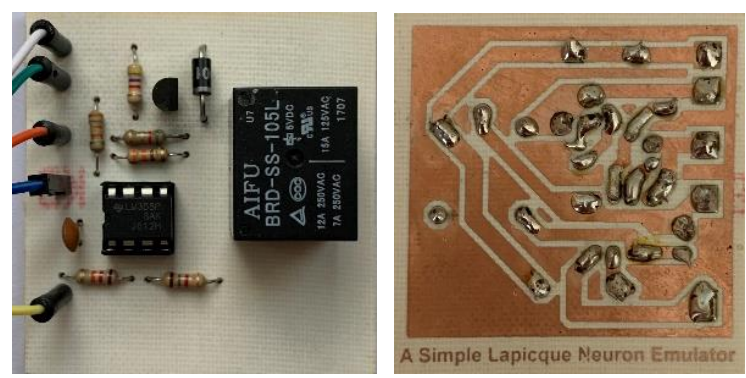

Fig. 2: Photo of the assembled Lapique neuron emulator circuit.

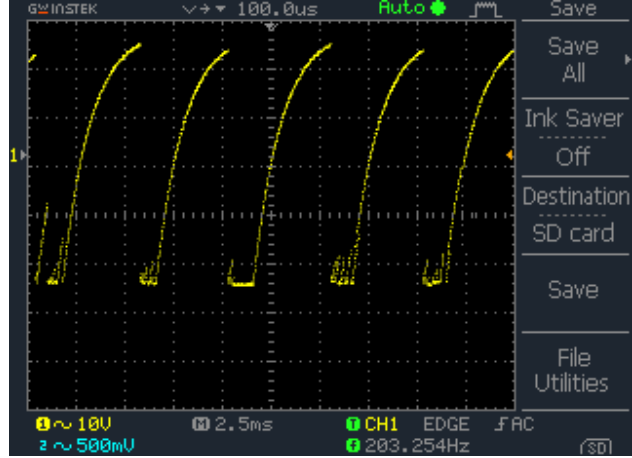

(a)

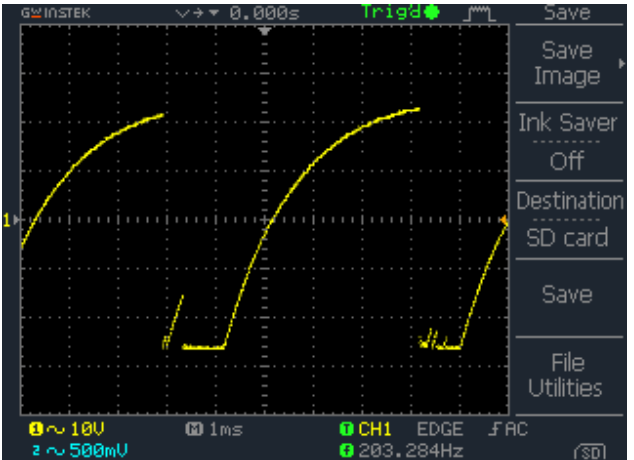

(b)

Fig. 3: (a) Lapique neuron emulator voltage. (b) Its zoomed view. 


\section{Conclusion}

Lapique neuron emulator is the simplest neuron model but it is still used in neuronal studiesdue to its simplicity and ability to mimic real neurons. In this study, it is shown that Lapique neuron emulator can be made using cheap off-the shelf components easily. The Lapique neuron emulator circuit we suggest in this paper is easy to build. Performance of Lapique neuron emulator is verified experimentally. It can emulate not only the successful but also the failed neuron firings. In this study, an electromechanical relay is used. If a solid-state relay or a semiconductor switch is used, the circuit can operate with higher firing (operating) frequencies. The emulator or its varieties can be easily made as graduate project by the senior students. A few of them can be connected to show phenomenon such as synchronization. That's why the emulator can be used in biomedical engineering education and research to show and prove neuronal concepts.

\section{References}

[1] Lapicque, L. (1907). Recherches quantitatives sur l'excitation electrique des nerfs traitee comme une polarization. Journal de Physiologie et de Pathologie Generalej, 9, 620-635.

[2] Brunel, N., \& Van Rossum, M. C. (2007). Lapicque's 1907 paper: from frogs to integrate-and-fire. Biological cybernetics, 97(5-6), 337-339.

[3] Hodgkin, A. L., \& Huxley, A. F. (1952). A quantitative description of membrane current and its application to conduction and excitation in nerve. The Journal of physiology, 117(4), 500-544.

[4] FitzHugh, R. (1955). Mathematical models of threshold phenomena in the nerve membrane. The bulletin of mathematical biophysics, 17(4), 257-278.

[5] FitzHugh, R. (1961). Impulses and physiological states in theoretical models of nerve membrane. Biophysical journal, 1(6), 445-466.

[6] Nagumo, J., Arimoto, S., \& Yoshizawa, S. (1962). An active pulse transmission line simulating nerve axon. Proceedings of the IRE, 50(10), 2061-2070.

[7] FitzHugh, R. (1969). Mathematical models of excitation and propagation in nerve. Biological engineering, 1(9), 1-85.

[8] Izhikevich, E. M. (2003). Simple model of spiking neurons. IEEE Transactions on neural networks, 14(6), 15691572 .

[9] Izhikevich, E. M. (2004). Which model to use for cortical spiking neurons?. IEEE transactions on neural networks, 15(5), 1063-1070.

[10] Izhikevich, E. M., \& Hoppensteadt, F. (2004). Classification of bursting mappings. International Journal of Bifurcation and Chaos, 14(11), 3847-3854.

[11] Izhikevich, E. M., Gally, J. A., \& Edelman, G. M. (2004). Spike-timing dynamics of neuronal groups. Cerebral cortex, 14(8), 933-944.

[12] Andrew, A. M. (2003). Spiking neuron models: Single neurons, populations, plasticity. Kybernetes, 32(7/8).

[13] Dayan, P., \& Abbott, L. F. (2001). Theoretical neuroscience (Vol. 806). Cambridge, MA: MIT Press.

[14] Gerstner, W., Kistler, W. M., Naud, R., \& Paninski, L. (2014). Neuronal dynamics: From single neurons to networks and models of cognition. Cambridge University Press.

[15] Mishra, A., \& Majhi, S. K. (2019). A comprehensive survey of recent developments in neuronal communication and computational neuroscience. Journal of Industrial Information Integration, 13, 40-54.

[16] Hayashi, T., Tokihiro, T., Kurihara, H., Nomura, F., \& Yasuda, K. (2018). Integrate and fire model with refractory period for synchronization of two cardiomyocytes. Journal of theoretical biology, 437, 141-148.

[17] Borges, F. S., Protachevicz, P. R., Lameu, E. L., Bonetti, R. C., Iarosz, K. C., Caldas, I. L., ... \& Batista, A. M. (2017). Synchronised firing patterns in a random network of adaptive exponential integrate-and fire neuron model. Neural Networks, 90, 1-7.

[18] Teka, W. W., Upadhyay, R. K., \& Mondal, A. (2017). Fractional-order leaky integrate-and-fire model with long-term memory and power law dynamics. Neural Networks, 93, 110-125.

[19] Tsigkri-DeSmedt, N. D., Hizanidis, J., Schöll, E., Hövel, P., \& Provata, A. (2017). Chimeras in leaky integrate-andfire neural networks: effects of reflecting connectivities. The European Physical Journal B, 90(7), 139. 
[20] Fei Chen \& Yuan-Ting Zhang ( 2010 ). A variant of the integrate-and-fire model to simulate the adaptive neural firing pattern . Biomedical Signal Processing and Control , 5, 66 - 69 .

[21] M.U. Akhmet ( 2012 ). Self-synchronization of the integrate-and-fire pacemaker model with continuous couplings. Nonlinear Analysis: Hybrid Systems , 6, 730 - 740.

[22] Marcos G. Quiles, Liang Zhao, Fabricio A. Breve \& Roseli A.F. Romero ( 2009 ). A network of integrate and fire neurons for visual selection. Neurocomputing, $72,2198-2208$.

[23] Jianfeng Feng ( 1999 ). Origin of firing varibility of the integrate-and-fire model . Neurocomputing , 26-27 , 117 122.

[24] Chris Christodoulou ( 2002 ). On the firing variability of the integrate-and-fire neurons with partial reset in the presence of inhibition . Neurocomputing, 44-46, 81 - 84 .

[25] Michele Bezzi, Thierry Nieus, Olivier J.-M. Coenen \& Egidio D’Angelo ( 2004 ). An integrate-and-fire model of a cerebellar granule cell . Neurocomputing , 58-60, 593 - 598.

[26] Maurice J. Chacron, Andre Longtin \& Khashayar Pakdaman ( 2004 ). Chaotic firing in the sinusoidally forced leaky integrate-and-fire model with threshold fatigue . Physica D: Nonlinear Phenomena , 192 , 138 - 160.

[27] Abulhair Saparov \& Michael A. Schwemmer ( 2015 ). Effects of passive dendritic tree properties on the firing dynamics of a leaky integrate-and-fire neuron. Mathematical Biosciences , 269 , 61 - 75 .

[28] Aleister Cachon \& Roberto A. V'azquez ( 2015 ). Tuning the parameters of an integrate and fire neuron via a genetic algorithm for solving pattern recognition problems . Neurocomputing , 148,187 - 197 .

[29] Junko Inoue \& Shinji Doi ( 2007 ). Sensitive dependence of the coefficient of variation of interspike intervals on the lower boundary of membrane potential for the leaky integrate-and-fire neuron model. Biosystems , 87 , 49 - 57 .

[30] Lubomir Kostal ( 2012 ). Approximate information capacity of the perfect integrate-and-fire neuron using the temporal code. Brain Research , 1434 , 136 - 141.

[31] A. Yadav, D. Mishra, R.N. Yadav, S. Ray \& P.K. Kalra ( 2007 ). Timeseries prediction with single integrate-and-fire neuron . Applied Soft Computing , 7, 739 - 745.

[32] Yener, Ş. Ç., \& Mutlu, R. (2019, November). A Microcontroller Implementation Of Hindmarsh-Rose Neuron ModelBased Biological Central Pattern Generator. In 2019 1st International Informatics and Software Engineering Conference (UBMYK) (pp. 1-4). IEEE.

[33] Sanchez-Sinencio, E., \& Linares-Barranco, B. (1989, August). Circuit implementation of neural FitzHugh-Nagumo equations. In Circuits and Systems, 1989., Proceedings of the 32nd Midwest Symposium on (pp. 244-247). IEEE.

[34] Linares-Barranco, B. E. R. N. A. B. E., Sánchez-Sinencio, E., Rodríguez-Vázquez, A., \& Huertas, J. L. (1991). A CMOS implementation of FitzHugh-Nagumo neuron model. IEEE Journal of Solid-State Circuits, 26(7), 956-965.

[35] Rajasekar, S., Murali, K., \& Lakshmanan, M. (1997). Control of chaos by nonfeedback methods in a simple electronic circuit system and the FitzHugh-Nagumo equation. Chaos, Solitons \& Fractals, 8(9), 1545-1558.

[36] Tamaševičiūtè, E., Mykolaitis, G., \& Tamaševičius, A. (2012). Analogue modelling an array of the FitzHugh-Nagumo oscillators. Nonlinear Analysis: Modelling and Control, 17(1), 118-125.

[37] Zhao, J., \& Kim, Y. B. (2007, August). Circuit implementation of FitzHugh-Nagumo neuron model using field programmable analog arrays. In Circuits and Systems, 2007. MWSCAS 2007. 50th Midwest Symposium on (pp. 772775). IEEE.

[38] http://people.ece.cornell.edu/land/PROJECTS/NeuralModels/

[39] Petrovas, A., Lisauskas, S., \& Slepikas, A. (2012). Electronic model of fitzhugh-nagumo neuron. Elektronika Ir Elektrotechnika, 122(6), 117-120.

[40] Tamaševicius, A., Bumeliene, S., Tamaševiciute, E., Mykolaitis, G., \& Kirvaitis, R. (2010, May). Desynchronization of mean-field coupled oscillators by remote virtual grounding. In Proc. of the 18th Int. Workshop on Nonlinear Dynamics of Electronic Systems (pp. 26-28). 